\title{
BMJ Open Qualitative findings on building a partnered approach to implementation of a group-based diabetes intervention in VA primary care
}

\author{
Jennifer Arney, ${ }^{1,2,3}$ Kimberly Thurman, ${ }^{1}$ Lindsey Jones, ${ }^{1}$ Lea Kiefer, ${ }^{1,3}$ \\ Natalie E Hundt, ${ }^{1,3}$ Aanand D Naik, ${ }^{1,3}$ LeChauncy D Woodard ${ }^{1,3}$
}

To cite: Arney J, Thurman K, Jones L, et al. Qualitative findings on building a partnered approach to implementation of a groupbased diabetes intervention in VA primary care. BMJ Open 2018;8:e018093. doi:10.1136/ bmjopen-2017-018093

- Prepublication history for this paper is available online. To view these files, please visit the journal online (http://dx.doi org/10.1136/bmjopen-2017018093).

Received 7 June 2017 Revised 15 November 2017 Accepted 17 November 2017

Check for updates

${ }^{1}$ VA HSR\&D Center for Innovations in Quality, Effectiveness and Safety (IQuESt), Michael E DeBakey Veterans Affairs Medical Center, Houston, Texas, USA

${ }^{2}$ Department of Sociology, University of Houston-Clear Lake, Houston, Texas, USA

${ }^{3}$ Baylor College of Medicine, Houston, Texas, USA

Correspondence to Dr LeChauncy D Woodard; Iwoodard@bcm.edu

\section{ABSTRACT}

Objective Conduct a formative evaluation to inform the implementation of 'Empowering Patients in Chronic Care' (EPIC), an evidence-based interdisciplinary group medical appointment intervention to improve collaborative goalsetting in patients with treated but uncontrolled diabetes. Design The formative evaluation involved qualitative, in-depth interviews with clinicians, structured according to the Promoting Action on Research in Health Services framework. Interviews elicited (1) participants' knowledge regarding interdisciplinary group self-management and goal-setting programmes and how well clinicians embrace these interventions (evidence), (2) physical and social climate at each target facility and how the intervention can best be embedded into routine primary care (context) and (3) site-specific needs to be addressed by our implementation team and clinicians' preparedness and intentions to participate in the intervention (facilitation). Setting Clinicians were part of a primary care setting at one of five participating medical facilities within one Veterans Health Administration Veterans Affairs regional network.

Participants We interviewed a snowball sample of 35 interdisciplinary clinicians engaged in diabetes management, practising leadership and administrators at target sites.

Results Most participants had previous experience with diabetes group self-management programmes and viewed group appointments as an effective approach to enhancing care. Discussions about existing group appointments provided a context for evaluating potential barriers and facilitators to implementing EPIC into target sites. Interviews revealed clinicians' expectations about the roles they would play in the intervention, their assessments of the roles and strategies to facilitate their performance in those roles.

Conclusions Successful implementation of evidencebased practices into routine care requires a partnered approach with engaged local staff. The intervention should address local goals and research objectives to encourage bidirectional engagement. Robust partnerships are nurtured further by sustained, open communication and must consider the context, target population and local experience to address barriers and facilitators to implementation.

\section{Strengths and limitations of this study}

- The study identified strategies for overcoming barriers to Empowering Patients in Chronic Care (EPIC) implementation, and outlines findings that can facilitate implementation of other evidencebased practices.

- We used clinicans' feedback to improve our programme by decreasing the amount of information presented in each session, increasing the number of sessions, and simplifying patient reading materials, and we used existing features, such as the group appointment mechanism, existing diabetes care teams and meeting spaces to implement our programme.

- We identified staff training and support needs and offered a variety of support strategies including cross-facility consultations and feedback on audiorecorded EPIC sessions.

- The snowball sampling technique may have led to recruitment of like-minded individuals.

- Although the study is a multisite randomised control trial, the data are from five veterans administration sites across the USA, and therefore, findings may not be directly applicable to other healthcare systems.

\section{INTRODUCTION}

Implementation and maintenance of evidence-based practices into primary care can involve numerous logistical and institutional challenges. Due to the collaborative nature of group medical appointments involving interdisciplinary providers, implementation of such an interdisciplinary intervention can be particularly challenging. We draw from literature on implementation of evidencebased practices to guide our implementation of an interdisciplinary group medical appointment intervention to improve collaborative goal-setting in patients with treated but uncontrolled diabetes. Much of the literature points to the importance of establishing strategic partnerships at intervention 
sites. From their experiences conducting a clinical trial of a telehealth intervention, Naik and colleagues ${ }^{1}$ identified strategies to aid timely translation of health services research into primary care; their recommendations highlight the importance of developing and sustaining strategic partnerships between clinical stakeholders and researchers. They recommend establishing clinical-research partnerships early in the implementation process and using them to facilitate allocation of resources, space and professional effort. ${ }^{1}$ Partners are vital when finalising intervention materials to ensure appropriateness for local populations, maximise collaboration and advance the goals of the clinical interventionists. Partnerships should endure for the course of the intervention and beyond; active communication and information sharing is essential to implementation success. Use of these strategies may also ensure sustainability and broader dissemination of the intervention. ${ }^{2}$

Drawing from these lessons, we conducted a formative evaluation of five hospital-based and community-based primary care clinics within one regional Veterans Affairs (VA) network. The evaluation sought to inform the implementation of an evidence-based, diabetes group intervention into routine primary care and to develop meaningful operational partnerships to promote continuation, dissemination and institutionalisation of the intervention. We applied the Promoting Action on Research in Health Services (PARIHS) implementation framework to guide data collection. ${ }^{3}$ Our findings detail the context of the target implementation sites, challenges that emerged, our responses to those challenges and partnerships that allowed us to identify and overcome implementation barriers.

\section{Rationale for the intervention}

Diabetes mellitus affects one in five veterans who use the VA healthcare system. Self-management skills are critical for controlling diabetes, ${ }^{45}$ but delivery of self-management support can be difficult to integrate into routine primary care. ${ }^{6}$ Effective self-management support involves collaborative goal-setting, discussions about daily actions to realise goals and ongoing provider-patient communication. ${ }^{78}$ Group visits can be an effective and efficient way for providers to deliver patient education and teach integral self-management strategies for chronic conditions such as diabetes. ${ }^{9}$

Drawing from the self-management literature, Naik and colleagues ${ }^{10}$ developed and tested 'Empowering Patients in Chronic Care' (EPIC), a group-based intervention to aid patients in setting personalised goals for diabetes control. The original EPIC intervention was delivered by research staff and designed to occur over four sessions among a group of 5-7 veterans (figure 1). Session 1 examined the importance of the diabetes ABCs (haemoglobin (Hb) A1c, blood pressure, cholesterol) and their role in providing a 'diabetes forecast' to aid self-management. Session 2 focused on the relationship between goals and action plans, as patients learnt how setting high-quality goals improves their ability to control diabetes. Session 3 introduced principles to encourage open communication and active participation with providers. Session 4 encouraged veterans to stay committed to their goals and action plans and assisted them with overcoming barriers to progress. Each session was followed by a brief, one-on-one coaching discussion about the veteran's goal(s). Patient participants reported an increase in diabetes self-efficacy and experienced greater $\mathrm{HbA} 1 \mathrm{c}$ control postintervention and sustained over time versus a comparison intervention. ${ }^{10}$

Based on the success of this efficacy study, we employed a partnered research approach to assess the effectiveness of EPIC after implementation into routine care in five primary care sites. The current trial was funded as part of the VA's Collaborative Research to Enhance and Advance Transformation and Excellence (CREATE) initiative, which promotes collaboration between research and clinical partners to improve veteran care. ${ }^{11}$ To facilitate collaboration with our clinical partners, our team modified EPIC to incorporate Patient-Aligned Care Teams (PACTs), the VA's model for the patient-centred medical home, and to include other primary care disciplines who deliver diabetes care. We integrated patient-reported levels of functional health literacy and activation to personalise communication between patients and clinicians and to enhance the intervention's effectiveness. In doing so, we moved EPIC from efficacy to implementation research within routine primary care.

\section{Implementation framework}

A formative evaluation of targeted sites using the PARIHS framework was conducted to facilitate implementation. PARIHS is a broad framework used to design and implement changes in evidence-based practice. It consists of three elements-evidence, context and facilitation-that guide the successful implementation of a health services intervention. ${ }^{12}$ The PARIHS framework was modified and elaborated by Stetler $e^{2} \mathrm{al}^{13}$ to address barriers and limitations in VA implementation research. Stetler's Guide was instrumental in executing our formative evaluation.

\section{METHODS \\ Data}

Using an interview guide aligned with the PARIHS framework, we conducted in-depth, qualitative interviews from a snowball sample of clinicians engaged in diabetes management (dietitians, nurses, educators, psychologists, primary care physicians and pharmacists) and practising leadership and administrators at each of our target sites (table 1). Feedback on the interview guide was elicited from key partners, and recommended changes were made prior to initiating interviews. Individuals were approached to solicit participation through email, followed by phone contact, with 90 approached in total. Of those approached, 35 participated in the 


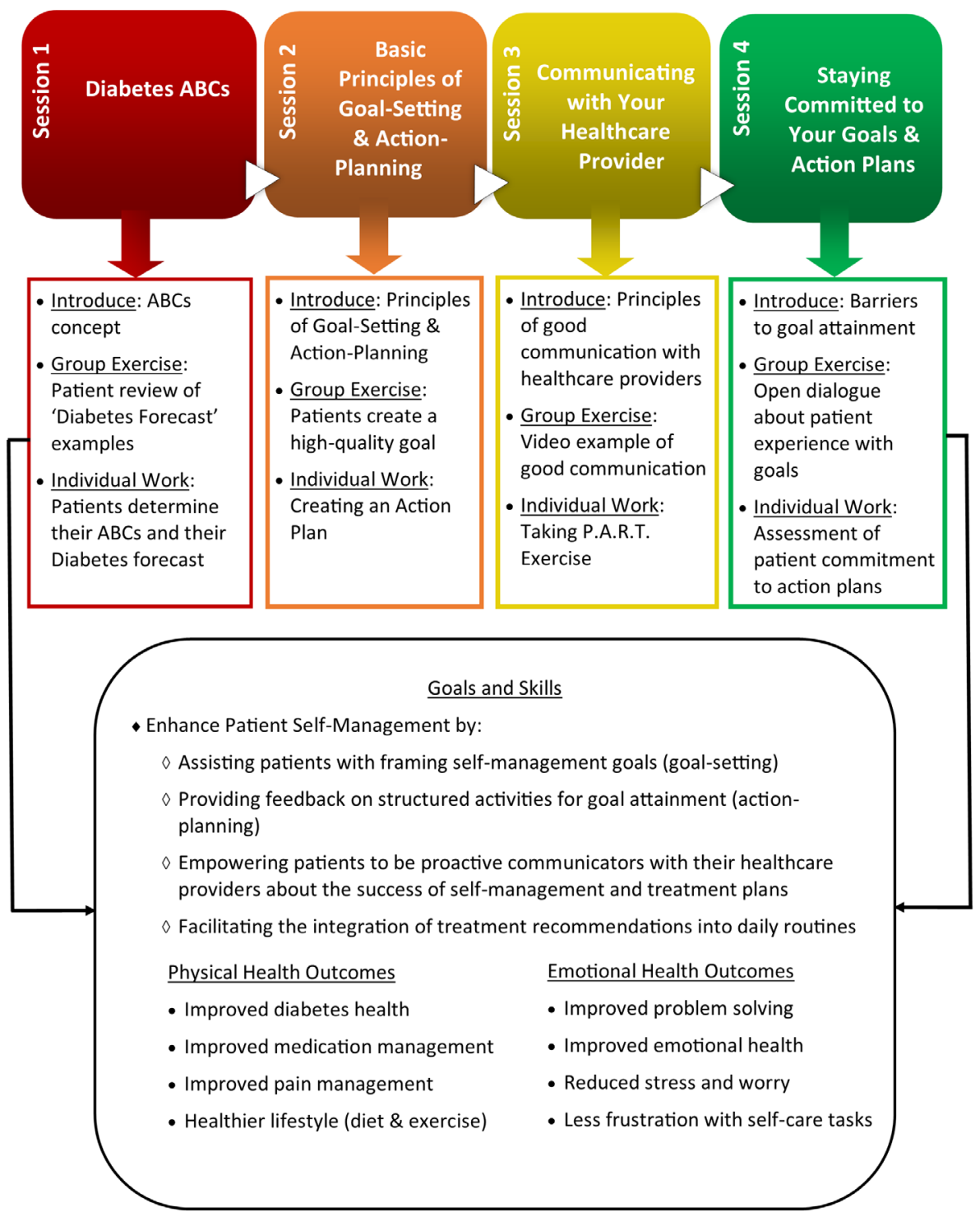

Figure 1 The four sessions of the original EPIC intervention. ABC, haemoglobin A1c, blood pressure, cholesterol; EPIC, Empowering Patients in Chronic Care.

study. Reasons for choosing not to participate were not recorded.

Participants were asked about (1) their knowledge regarding group self-management programme and how well primary care clinicians embrace our training/fidelity programme for personalised goal-setting (evidence), (2) physical and social climate at each target facility and how the intervention can best be embedded locally into routine primary care (context) and (3) site-specific needs to be addressed by our implementation team and clinicians' preparedness and intentions to participate in the intervention (facilitation) (table 2).
Interviews were conducted by telephone from June 2014 to January 2015 by authors JA or KT, two female medical sociologists experienced with qualitative interviewing. They lasted roughly $45-60 \mathrm{~min}$ and were digitally recorded, transcribed and analysed. Field notes were made during and after interviews. Transcripts were not returned to participants for review, and no repeat interviews were conducted. No relationship was established between participants and interviewers prior to the start of the study. Names and academic degrees of interviewers were included in the initial recruitment email sent to participants. No other characteristics about the 
interviewers were disclosed to participants. Prior to interviews, participants were informed about the EPIC intervention and the goals of the interview.

\section{Analysis}

Analysis was guided by principles of framework analysis, which allow themes to develop both from a prior framework (eg, PARIHS) and the narratives of research participants. ${ }^{14}$ To harness an initial coding scheme, two coders independently coded five transcripts, then met to discuss discrepancies. The resulting coding scheme was applied to all 35 transcripts in Atlas.ti. The coders frequently met to discuss new codes and to resolve discrepancies. Next, coders identified commonalities and divergent themes within and across participant categories. Finally, coders charted themes across participant subgroups to identify meaningful associations and patterns in the data. Coding centred on themes consistent with the PARIHS framework: evidence, context and facilitation. Subthemes were identified within each of the major domains, as reflected in the Results section below. Sample size was determined by thematic saturation, defined a priori as occurring when two independent coders identified no new codes on three consecutive transcripts. ${ }^{15}{ }^{16}$ Our snowball sampling technique allowed us to reach data saturation at the site level among primary care and clinic leadership personnel involved in the intervention implementation. Our intent was not to reach saturation with each healthcare profession involved in the intervention processes, as that was not the study aim. We sought to understand the range
Table 1 Number of participants by specialisation at target sites

Site 1 Site 2 Site 3 Site 4 Site 5 Total

\begin{tabular}{lrrrrrr}
\hline $\begin{array}{l}\text { Behavioural } \\
\text { health staff }\end{array}$ & 3 & 5 & 0 & 2 & 1 & 11 \\
$\begin{array}{l}\text { Patient-aligned } \\
\text { care team } \\
\text { members }\end{array}$ & 4 & 5 & 1 & 5 & 3 & 18 \\
$\begin{array}{l}\text { Clinical practice } \\
\text { leadership }\end{array}$ & 0 & 2 & 0 & 1 & 0 & 3 \\
\hline $\begin{array}{l}\text { Administrators } \\
\text { Totals }\end{array}$ & 0 & 2 & 1 & 0 & 0 & 3 \\
\hline
\end{tabular}

of implementation needs and barriers across all target implementation sites. Participants provided feedback on findings for use during a subsequent trial of the EPIC intervention.

All respondents provided verbal consent prior to participating in the interview.

\section{RESULTS}

We interviewed 35 clinicians, clinical practice leadership and administrators at our target sites (table 1). The sample included 28 women; 29 were white/Caucasian, 5 were Asian and 1 was African-American. Analysis provided insight into aspects of evidence, context and facilitation regarding implementation of our intervention.

Table 2 PARIHS framework and related interview questions

\begin{tabular}{lll}
\hline Domain & Description & Related interview questions \\
\hline Evidence & Knowledge of group self-management & How much of a priority is diabetes care at VAMC? \\
& programmes; how well clinicians & How great is the need to improve diabetes care in this VISN? \\
& embrace our programme. & What factors encourage/deter providers to hold group appointments? \\
& What factors encourage/deter patients' involvement in group \\
& appointments?
\end{tabular}

Context Physical and social climate at each site; how EPIC can be best embedded into routine workflows.
Please tell me about a typical SMA at this facility.

How are PACTs involved in the shared medical appointment?

How are patients recruited and scheduled to take part in the SMA?

How many patients are scheduled to attend an SMA? How many show up?

Please tell me about the current diabetes initiatives at your facility. How does EPIC fit with the current diabetes initiatives?

$\begin{array}{ll}\text { Facilitation } & \begin{array}{l}\text { Clinicians' interest and availability to } \\ \text { assist with EPIC; site-specific needs to }\end{array} \\ \text { address. } & \text { Wow do you imagine your role in EPIC? } \\ & \text { What could be done to help you in this role? } \\ & \text { Do you think you will be performing additional work? } \\ & \text { What tasks do you think you will be responsible for? } \\ & \text { How can we make this more efficient? }\end{array}$

EPIC, Empowering Patients in Chronic Care; PACT, Patient-Aligned Care Team; PARIHS, Promoting Action on Research in Health Service; SMA, shared medical appointment; VAMC, Veterans Affairs Medical Center; VISN, Veterans Integrated Service Network. 
Table 3 Guidelines to facilitate implementation of an evidence-based health services intervention

\begin{tabular}{|c|c|c|}
\hline Guideline & Description & Examples \\
\hline $\begin{array}{l}\text { Draw from local } \\
\text { objectives and } \\
\text { experience }\end{array}$ & $\begin{array}{l}\text { Local objectives and experience help anticipate } \\
\text { barriers and facilitators. Understanding how the } \\
\text { intervention can serve local clinical and leadership } \\
\text { goals should guide implementation. Align local } \\
\text { objectives and research aims to encourage } \\
\text { robust bidirectional engagement to sustain strong } \\
\text { partnerships and compel progress. Draw on the } \\
\text { pursuit of these symbiotic goals when clinicians } \\
\text { view the intervention as redundant to ongoing } \\
\text { efforts. }\end{array}$ & $\begin{array}{l}\text { We aligned research with the network's local } \\
\text { Hypoglycaemia Safety Initiative which seeks to } \\
\text { establish shared diabetes goals between patients } \\
\text { and providers. We also incorporated a locally } \\
\text { produced Speak Up! video into Session } 4 \text { which } \\
\text { addressed patient-provider communication. }\end{array}$ \\
\hline $\begin{array}{l}\text { Tailor the } \\
\text { intervention to the } \\
\text { context }\end{array}$ & $\begin{array}{l}\text { Learn about existing structures and contextual } \\
\text { features that could aid implementation. }\end{array}$ & $\begin{array}{l}\text { We used the group medical appointment to } \\
\text { structure the intervention to address workflow } \\
\text { concerns by allowing clinicians workload credit } \\
\text { for participation. We built supportive relationships } \\
\text { with primary care clinicians to foster a desired } \\
\text { collaborative culture and reduce perceived barriers } \\
\text { to collaboration within primary care. }\end{array}$ \\
\hline $\begin{array}{l}\text { Consider the target } \\
\text { population }\end{array}$ & $\begin{array}{l}\text { Tailor the intervention accordingly to the local } \\
\text { patient population. }\end{array}$ & $\begin{array}{l}\text { We adjusted the number of sessions from four to } \\
\text { six and adapted program materials to address poor } \\
\text { health literacy. }\end{array}$ \\
\hline $\begin{array}{l}\text { Anticipate and } \\
\text { plan for barriers to } \\
\text { implementation }\end{array}$ & $\begin{array}{l}\text { Identify facility-level barriers early and develop } \\
\text { appropriate strategies to facilitate implementation. } \\
\text { Implementation will require strategic multilevel } \\
\text { partnerships and time to process resolutions } \\
\text { locally. Affording partners time to address barriers } \\
\text { will enhance implementation efforts and deepen } \\
\text { partnerships. }\end{array}$ & $\begin{array}{l}\text { At some sites, we identified a shortage of available } \\
\text { pharmacists for participation. In response, we } \\
\text { adjusted pharmacy participation to occur at every } \\
\text { other visit and allowed telephone follow-up visits. } \\
\text { We also identified a lack of capacity in some of the } \\
\text { local phlebotomy laboratories for baseline blood } \\
\text { draws and worked with clinics to schedule these in } \\
\text { a staggered manner. }\end{array}$ \\
\hline $\begin{array}{l}\text { Provide ongoing } \\
\text { support }\end{array}$ & $\begin{array}{l}\text { Elicit and satisfy local training needs. Provide } \\
\text { avenues for communication with the research team. }\end{array}$ & $\begin{array}{l}\text { We conducted EPIC-specific training requested by } \\
\text { clinicians prior to implementation; offered cross- } \\
\text { facility consultations so staff at various sites could } \\
\text { learn from one another and provided feedback on } \\
\text { audio-recorded group appointments. }\end{array}$ \\
\hline
\end{tabular}

EPIC, Empowering Patients in Chronic Care.

\section{Evidence}

Interviews elicited knowledge regarding group self-management programmes and the degree to which participants embraced key components of EPIC. Most clinicians had previous experience with diabetes group self-management programmes or medical appointments and viewed group medical appointments as effective for enhancing care. Discussions about existing group medical appointments provided a context for evaluating potential barriers and facilitators to implementing EPIC into target sites.

\section{Status of diabetes care}

A large demand for diabetes services seemed consistent across facilities. Multiple facilities created group medical appointments to address the high volume of patients with poorly controlled diabetes. According to one participant, a diabetes education coordinator and registered nurse,

We have over three thousand patients right now active in our [diabetes] program. I have a clinical nurse specialist right now working with three or four endocrinologists seeing these patients.
When asked how well facilities are doing in managing diabetes patients, clinicians often indicated that they do the best they can, given available resources. In particular, clinicians remarked that they were not staffed to provide personalised diabetes self-care support. Another participant, a registered nurse, discussed the ongoing programme at her facility and shared how it could be improved with more staffing:

The basic initial group is partially didactic... it's not as interactive as it could be if we had more help... It's not set up to do like a medical appointment where we'll... have that patient's one-on-one follow up... Again there isn't the staffing.

The EPIC intervention could feasibly mitigate such staffing problems by assigning 2-3 clinicians protected time to work with patients with diabetes in a group medical appointment.

Many clinicians acknowledged that diabetes care could be improved at their facility. Clinicians often referred to the utility of a diabetes group medical appointment 
in improving diabetes care. According to a clinical pharmacist,

We need to do better with managing our diabetic patients... Since our providers have large patient panels... I think if they're able to set aside a half day a month [to hold group medical appointments]... they could target more of their patients all at once.

This suggests that some clinicians value the group medical appointment model and perceive a need for an intervention such as EPIC. Other clinicians described ongoing diabetes initiatives, but still acknowledged a need for additional effort. According to a nurse practitioner,

I think we still have a large population that's uncontrolled, despite all of our efforts.

This suggests that these sites could benefit from programmes providing more tailored diabetes self-management support. Our modified EPIC intervention extends beyond traditional diabetes care by incorporating collaborative goal-setting and personalised health information to fine-tune the goal-setting process.

\section{Objectives and rationale for group medical appointments}

Clinicians generally felt positive about group programmes and medical appointments and reported benefits of the group dynamic. Many clinicians noted a broad range of goals for group medical appointments or group self-management programmes. One clinical pharmacist viewed the group setting as an opportunity to engage with patients motivationally. She said,

It's about empowering the patient and educating the patient, debunking myths... just having strategic conversations with the patients.

Some clinicians valued the peer support afforded by group appointments. According to a registered dietician,

The biggest part is just that they get to kind of feed off of each other and they talk about what works and what doesn't... I think that the fact that they can help teach each other is most important.

Suggestions clinicians shared about aspects of group medical appointments that work best for diabetes care were incorporated into the intervention (see below). Groups could most effectively be led by a diabetes specialist with a background in motivational interviewing and behavioural health. Nutrition experts and pharmacists would be vital, as veterans' goals were expected to centre often on diet and medication management.

\section{Patient-level barriers and facilitators}

From personal experience, clinicians discussed how patients react to group appointments. Patients face several barriers to participation including transportation, other commitments, low perceived value, language barriers, reluctance to take part in a group, privacy concerns and reluctance to disclose medical history and health complaints. One clinical pharmacist believed that veterans may simply be 'burnt out on didactic learning'. Clinicians identified several facilitators to patient participation in group appointments including the invitation to bring a family member or friend, the VA transportation service, a clear understanding of what to expect, the opportunity to benefit from additional care and the opportunity to connect and learn from other veterans. Some acknowledged that patients would be interested to learn practical skills for diabetes self-management. One participant, a registered nurse, noted that patients would appreciate taking part in EPIC's interactive format; veterans would enjoy 'knowledge gained from information provided in a different manner.' We operationalised many of these facilitators into the intervention design. Veterans were encouraged to bring supportive family. A recruitment script was developed to deliver a clear understanding of the group and emphasise the opportunity to serve and learn from other veterans. To facilitate peer support, group membership was consistent between sessions.

\section{Context}

Contextual features of each target site included characteristics of the local patient population, which prompted the team to re-evaluate the intensity of the intervention. Clinicians' perceptions of the intervention and logistical concerns also influenced implementation.

\section{Low health literacy population}

An early contextual issue was the health literacy level of the target population. After reviewing our intervention materials, clinicians at one site suggested that the veterans would not be able to master the quantity of information over the span of four sessions. According to a clinical psychologist,

A lot of the Veterans we work with lead rough lives, have unstable housing, many are homeless or just kind of in transitional housing. So I think... to have to get through all of this in one session, I think that would be just very difficult.

Given the group structure, facilitators would be required to spend additional time explaining unfamiliar concepts and engaging patients in the material. From this and similar feedback, we expanded EPIC from four to six sessions.

\section{Challenges to interdisciplinary collaboration}

Interdisciplinary collaboration is vital to help patients set and achieve personalised goals in chronic disease management. Some clinicians-patient educators and nurses-discussed successful collaborations at their sites as occurring primarily among people of the same disciplines and not often involving primary care providers. Clinicians referred to a lack of interdisciplinary practice and alluded to the benefits of a more interdisciplinary model of diabetes care. The group medical appointment 
was specifically discussed as a way to 'get a lot of work done' by manualising interdisciplinary collaboration. According to a registered dietician,

You know, the one thing we keep trying to push to them [to encourage group appointments] is like, 'you're really- you're getting a lot of work done in a short time.' We definitely have some that are of the old school; it's appointment time, one patient at a time, let me take care of this patient and move on to the next. So we do have resistance to [group appointments], but we keep trying to put that out there. You really can get a lot of work done in a short time if you would just open your eyes and be willing to try this.

This suggests clinician support for the group appointment model as a means to improve access to care. Other challenges to interdisciplinary collaboration include coordination of schedules and underutilisation of specialists by primary care clinicians. In response, we developed strategic operational partnerships to encourage multilevel support for the group appointment model and maximise utilisation of existing personnel.

\section{Fit with existing programmes}

Implementation of EPIC was suggested as optimal in clinics with a clear need-clinics that lack ongoing diabetes management resources. Alongside existing diabetes programmes, the intervention was sometimes seen as competition. As one clinical pharmacist indicated, "We're very proud of our program; we've worked very hard for it", suggesting that the intervention would threaten their existing programme. She said,

I think our program that we have here speaks to the fact that we are running a good show. We do have patients that we have to say, 'I'm sorry you've been here too long; you need to go.' We do have patients that call and say, 'Hey my friend said he went to this class; I want to go to that class too. It sounds really good.' And that happens more than I can tell you.

One clinical pharmacist questioned,

Why are we doing EPIC here at (facility)? Do we really need it? Like, is it better than what we're currently doing? Why is it so important? I think people would be supportive of it but... we've got to be convinced that this is something better than what we've been doing.

This clinician also inquired why the research team had chosen their site. This was related to a perception of oversight and a belief that leadership deemed their efforts as inefficacious. She asked,

Where is this EPIC coming from and why are we being tasked with making it happen here at (facility)?... Were we identified as being a facility that's not performing well with our A1Cs or something?
In contrast, clinicians at another site with no ongoing diabetes programmes welcomed the opportunity to collaborate with researchers and colleagues to implement the intervention into routine practice. According to a registered nurse,

We're all kind of looking forward to it. At least our close little group here that's been working in diabetes.

Thus, clinics without well-established diabetes self-management programmes may be the earliest adopters. However, the possibility of collaborating synergistically with other programmes is a rich opportunity for the intervention to occur alongside existing efforts.

\section{Logistical elements at the target sites}

Two facility-level barriers emerged as impediments to the group appointment model: inadequate staff and lack of time. Clinicians noted that they would have difficulty blocking off time to participate in the intervention. They believed that such an intervention would require additional work and may impact the national emphasis on minimising wait times for patient care. One licensed practical nurse shared,

With everything that's on the news right now about getting patients in within fourteen days... there is a lot of stress on the providers... That might be the only barrier for them attending these group appointments. I'm sure their priority is to make sure the patient is seen.

Interestingly, while the clinicians perceived a lack of support for group initiatives, individuals in leadership positions acknowledged their support of EPIC. According to an administrator and primary care physician,

I think speaking to the importance of research and teamwork, getting people together for the betterment of patient care and the collegial approach to doing the kind of thing that brings people from different disciplines together, particularly nursing and the primary care providers. I think that's where we've got to wear that cap to get the right people engaging and working together.

Space to host the groups was another concern. Four of the five facilities described space options, from a conference room to any number of patient education rooms. Only one facility consistently regarded space as a barrier to implementation. Relying on our partnerships, we overcame space concerns at that site with cooperative local assistance.

\section{Facilitation}

Interviews revealed clinicians' expectations about the roles they expected to play in the intervention, their assessments of the roles and strategies to facilitate their performance in those roles. 


\section{Training}

Many clinicians expected to participate in the EPIC groups and looked forward to doing so, but requested training on EPIC protocol and materials. They desired a better understanding of how the instructor manual corresponds with the patient workbook. They also desired clarification on how to transition the group meeting to individual coaching. While clinicians were comfortable with traditional didactic education groups, many implied that inclusion of collaborative goal-setting may require training in motivational interviewing. Many clinicians wished to view a simulated group. In response, we developed an accredited training protocol that included live vignettes and condensed the patient workbook into a 'teacher's edition' clinician manual. To assure training fidelity, we conducted audit and feedback after Session 1, monitored session quality and provided feedback on-demand throughout the trial.

\section{Communication}

Clinicians wished to be in close communication with the research team and the intervention teams at other sites. One participant, a diabetes education coordinator and registered nurse, desired "a network that I can go to, to ask questions... just knowing that I can get in touch with someone". One clinical pharmacist desired contact information for a "resource person to somebody [I can] contact to help with unexpected things". We provided points-of-contact for logistical research questions and created a practice-network for clinicians through quarterly, cross-facility calls.

\section{Assurance of leadership support}

Some clinicians requested that our research team act as a liaison to ensure leadership support for their involvement in EPIC. Specifically, one of the clinical pharmacist participants asked that our project staff " [get] supervisors to buy into it so that we have the time and the resources that we need to get the job done". A registered nurse also indicated that researchers could "push for administrative support' to allocate necessary implementation resources. From our strong operational partnerships (arising from the CREATE grant mechanism), we were able to establish local clinical leadership support for the intervention including time and effort for the study clinicians. To maintain frequent communication and strong partnerships, we hosted a steering committee meeting quarterly to discuss leadership support and engagement.

\section{DISCUSSION}

Analysis of interviews with 35 clinicians, clinical practice leadership and administrators revealed insight into aspects of evidence, context and facilitation regarding implementation of EPIC into five hospital-based and community-based primary care clinics within one regional VA network. Most respondents had experience with diabetes group education, self-management or group medical appointments and viewed the group appointment as a valuable mechanism for patient care. However, we learnt that contextual features, such as a low health literacy patient population, lack of resources and clinicians' competing demands, would require modifications to the intervention and additional support from the study team. We identified training needs (ie, training in motivational interviewing techniques) and participants' preferences for specific types of support to assist them throughout the intervention period.

We responded to information elicited in the formative evaluation interviews in several ways. We used the group medical appointment to address staffing and workload issues while creating an environment for staff to teach critical self-management skills. We incentivised training with Continuing Education Units to encourage fidelity to the intervention. Based on partner feedback, we tailored our training protocol to address common concerns and improve staff engagement. We acknowledged the needs of the local patient population with adaptations for low-health literacy. We developed strategic multilevel partnerships to ensure the mobilisation of necessary resources and broad support for the intervention. We supported EPIC clinicians with open communication to engage a shared purpose and effort in the implementation. Drawing from our data and the original PARIHS framework, we offer five guidelines to facilitate implementation of an evidence-based group-clinic model of a health services intervention (see table 3). Our formative evaluation revealed information that was used to facilitate the launch of EPIC into five remote implementation sites. While the specific information we gained was unique to the five sites we evaluated, a formative evaluation would be vital for any interdisciplinary team in any healthcare system interested in implementing a group behavioural health intervention.

Numerous theories, models and frameworks are available to facilitate implementation of evidencebased practices into routine care. ${ }^{17}$ The PARIHS framework has been widely applied and is celebrated for its flexibility, intuitive appeal and attention to the multifaceted context in which implementation occurs. ${ }^{12}$ Previous research has used the elements of PARHIS (evidence, context and facilitation) to interpret and map findings of assessment data aimed to facilitate implementation of evidence-based practices. ${ }^{12} \mathrm{We}$ extend this previous work by offering specific guidelines and examples that can inform future implementation efforts.

This study is limited in its focus on one closed healthcare system and may not be generalisable to sites outside of the Veterans Health Administration. An additional limitation may be our snowball sampling strategy, which precluded reaching thematic saturation across each professional specialisation. However, this technique allowed us to reach data saturation at the site level among primary 
care and clinic leadership personnel involved in the intervention implementation. Snowball sampling may result in recruiting like-minded individuals, which would limit the breadth of our findings. In spite of these limitations, our study provides key information to guide successful implementation of evidence-based practices into routine care. Importantly, such efforts require a partnered approach with engaged local staff. The intervention should address local goals and research objectives to encourage bidirectional engagement. Robust partnerships are nurtured further by sustained, open communication and must consider the context, target population and local experience to address barriers and facilitators to implementation.

Contributors JA, LK, ADN and LCDW contributed to the study design. JA and KT contributed to the data collection and analysis. JA, LK, NEH, LJ, ADN and LCDW contributed to the manuscript development. All authors contributed to the final revisions and approval of the version to be published and agreed to be accountable for all aspects of the work.

Funding This work was supported by a grant from the Department of Veterans Affairs, HSR\&D grant CRE 12-426 (PI: LW), partially supported by the facilities and resources of the VA HSR\&D Houston Center for Innovations in Quality, Effectiveness and Safety (CIN 13-413), Michael E DeBakey VA Medical Center, Houston, Texas, and partially supported by a Career Development Award (CDA 13-264) awarded to NEH.

Competing interests None declared.

Patient consent Obtained.

Ethics approval Baylor College of Medicine Institutional Review Board (H-33772).

Provenance and peer review Not commissioned; externally peer reviewed.

Data sharing statement № additional unpublished data are available.

Open Access This is an Open Access article distributed in accordance with the Creative Commons Attribution Non Commercial (CC BY-NC 4.0) license, which permits others to distribute, remix, adapt, build upon this work non-commercially, and license their derivative works on different terms, provided the original work is properly cited and the use is non-commercial. See: http://creativecommons.org/ licenses/by-nc/4.0/

(C) Article author(s) (or their employer(s) unless otherwise stated in the text of the article) 2018. All rights reserved. No commercial use is permitted unless otherwise expressly granted.

\section{REFERENCES}

1. Naik AD, Lawrence B, Kiefer $L$, et al. Building a primary care/research partnership: lessons learned from a telehealth intervention for diabetes and depression. Fam Pract 2015;32:216-23.

2. Scheirer MA, Dearing JW. An agenda for research on the sustainability of public health programs. Am J Public Health 2011;101:2059-67.

3. Stetler CB, Legro MW, Wallace CM, et al. The role of formative evaluation in implementation research and the QUERI experience. J Gen Intern Med 2006;21(Suppl 2):S1-8.

4. Bodenheimer T, Wagner EH, Grumbach K. Improving primary care for patients with chronic illness: the chronic care model, Part 2. JAMA 2002;288:1909-14.

5. Norris SL, Lau J, Smith SJ, et al. Self-management education for adults with type 2 diabetes: a meta-analysis of the effect on glycemic control. Diabetes Care 2002;25:1159-71.

6. Parchman ML, Zeber JE, Palmer RF. Participatory decision making, patient activation, medication adherence, and intermediate clinical outcomes in type 2 diabetes: a STARNet study. Ann Fam Med 2010;8:410-7.

7. Lorig K. Action planning: a call to action. J Am Board Fam Med 2006;19:324-5.

8. Naik AD, Kallen MA, Walder A, et al. Improving hypertension control in diabetes mellitus: the effects of collaborative and proactive health communication. Circulation 2008;117:1361-8.

9. Jaber R, Braksmajer A, Trilling J. Group visits for chronic illness care: models, benefits and challenges. Fam Pract Manag 2006;13:37-40.

10. Naik AD, Palmer N, Petersen NJ, et al. Comparative effectiveness of goal setting in diabetes mellitus group clinics: randomized clinical trial. Arch Intern Med 2011;171:453-9.

11. US Department of Veterans Affairs: Health Services Research \& Development. HSR\&D collaborative research to enhance and advance transformation and excellence (CREATE) initiative. USA: US Department of Veterans Affairs, 2016. http://www.hsrd.research.va. gov/centers/create (accessed 20 Mar 2017).

12. Helfrich CD, Damschroder LJ, Hagedorn HJ, et al. A critical synthesis of literature on the promoting action on research implementation in health services (PARIHS) framework. Implement Sci 2010;5:82.

13. Stetler CB, Damschroder LJ, Helfrich CD, et al. A Guide for applying a revised version of the PARIHS framework for implementation. Implement Sci 2011;6:99.

14. Ritchie J, Spencer L. Qualitative data analysis for applied policy research. In: Bryman A, Burgess RG, eds. Analyzing qualitative data. First Edn. New York: Routledge, 1994:173-94.

15. Morse JM. The significance of saturation. Qual Health Res 1995;5:147-9.

16. Aita VA, Mcllvain HE. An armchair adventure in case study research. In: Crabtree BF, Miller ML, eds. Doing qualitative research. Second Edn. London: Sage, 1999.

17. Nilsen P. Making sense of implementation theories, models and frameworks. Implement Sci 2015;10:53. 